Supplement of Atmos. Chem. Phys., 17, 4369-4385, 2017

http://www.atmos-chem-phys.net/17/4369/2017/

doi:10.5194/acp-17-4369-2017-supplement

(C) Author(s) 2017. CC Attribution 3.0 License.

(c) (i)

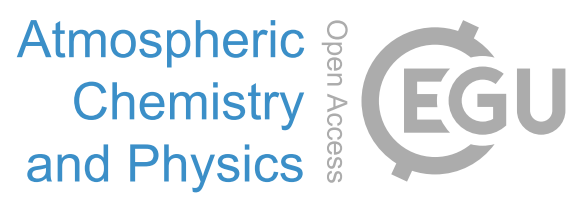

Supplement of

\title{
Hygroscopic properties of aminium sulfate aerosols
}

Grazia Rovelli et al.

Correspondence to: Jonathan P. Reid (j.p.reid@bristol.ac.uk)

The copyright of individual parts of the supplement might differ from the CC-BY 3.0 licence. 


\section{Supplementary Information outline:}

- Table S1: List and description of the uncertainties taken into account when calculating the hygroscopicity properties of aerosols from comparative kinetics experiments in a CK-EDB.

5 - Table S2: Measured densities and refractive indices of aminium sulphates solutions.

- Table S3: $3^{\text {rd }}$ order polynomial fitted parameters for densities, calculated melt densities and melt refractive indices calculated with molar refraction mixing rule for the six considered aminium sulphates.

- Table S4: Comparison of pure melt densities from this work and from Clegg et al. (2013) and solid densities from Qiu and Zhang (2012).

10 - Table S5: Coefficients of the fitted equations of the measured apparent molar volumes.

- Table S6: Extended ZSR fitted coefficients at two fixed water activities $\left(a_{w}, 0.80\right.$ and 0.925$)$ for mixtures of aminium sulphate salts and sulphuric acid.

- Figure S1: Schematics of the EDB experimental setup used in this work.

15 - Figure S2: Measured densities of aminium sulphates solutions and $3^{\text {rd }}$ order polynomial fittings of data and measured refractive indices as a function of mass fraction and the corresponding molar refraction mixing rule fitting.

- Figure S3: Comparison of measured $G F_{r}$ from CK-EDB experiments and $G F_{r}$ data from H-TDMA measurements by Qiu and Zhang (2012)

- $\quad$ Figure S4: Measured and fitted apparent molar volumes of aminium sulphates in aqueous solutions from this work and from Clegg et al. (2013). 
Table S1: List and description of the uncertainties taken into account when calculating the hygroscopicity properties of aerosols from comparative kinetics experiments in a CK-EDB.

\begin{tabular}{|c|c|c|}
\hline Quantity & Symbol/Formula & Uncertainties \\
\hline Water activity & $a_{w}$ & $\begin{array}{l}\text { - } a_{w} \text { : uncertainty from RH determination in the EDB } \\
\text { measurements (Davies et al., 2013): } \\
\text { - } \mathrm{RH}>80 \% \text { (pure water used as a probe): } \\
R H=R H_{w-\left(-0.020 R H_{w}+0.021\right)}+\left(0.169 R H_{w}{ }^{2}-0.04 R H_{w}+0.194\right) \\
\text { - } \mathrm{RH}<80 \%(\mathrm{NaCl} \text { used as a probe): } \\
R H=R H_{e q}+\left(-0.0175 R H_{e q}{ }^{2}-0.0005 R H_{e q}+0.017\right) \\
\quad-\left(-0.0266 R H_{e q}{ }^{2}+0.0086 R H_{e q}+0.017\right)\end{array}$ \\
\hline
\end{tabular}

- $a$ (measured): $\pm 100 \mathrm{~nm}$ (Davies, 2014), <1\% for droplets

$\begin{aligned} & \text { Radial growth } \\ & \text { factor }\end{aligned} \quad G F_{r}=\frac{a}{a_{d r y}}$
bigger than $10 \mu \mathrm{m} .{ }^{(a)}$

- $a_{d r y}$ : uncertainty on the initial radius extrapolation at $\mathrm{t}_{0}$ $\left(a_{d r y_{-100 \mathrm{~nm}}}^{+150 \mathrm{~nm}}\right)$; uncertainty on the initial solution concentration.

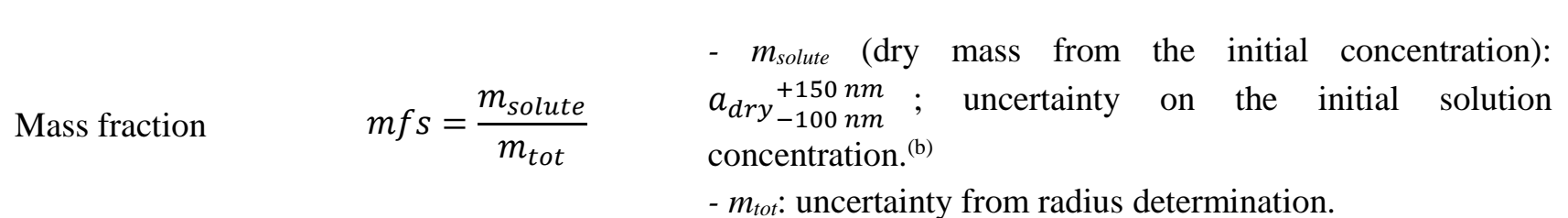

- $m_{t o t}$ : uncertainty from radius determination.

\begin{tabular}{|c|c|c|}
\hline Mole curves & $\frac{n_{\text {water }}}{n_{\text {solute }}}$ & $\begin{array}{l}\text { - } n_{\text {water }} \text {, calculated from } m_{\text {water }}=m_{\text {tot }}-m_{\text {solute }} \text { (see uncertainties } \\
\text { indicated above for } m f s \text { ). }\end{array}$ \\
\hline
\end{tabular}

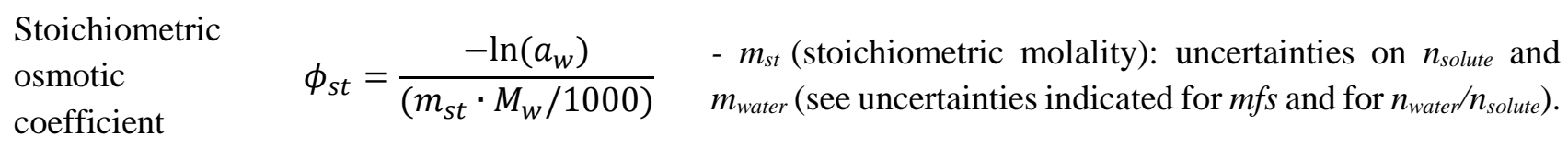

5 Symbols: $a_{w}$; water activity; $G F_{r}$ - radial growth factor; $a$-droplet radius; $a_{d r y}$ - dry particle radius; mfs - mass fraction of solute; $m_{\text {solute }}$ - mass of solute in the particle; $m_{t o t}$ - total particle mass; $n_{\text {water }}$ - moles of water; $n_{\text {solute }}$ - moles of solute; $\phi_{s t}-$ stoichiometric osmotic coefficients; $m_{s t}$ - stoichiometric molality; $M_{w}$ - molecular mass of water. 
Notes:

(a) The optical phase function measurements in the CK-EDB used to estimate the size of a levitated droplet are taken at a wavelength of $532 \mathrm{~nm}$; however, the wavelength dependence of refractive index was considered negligible (e.g. for pure water $m=1.333$ at $589 \mathrm{~nm}$ and $m=1.335$ at $532 \mathrm{~nm}$ ) and introduced negligible error in the determination of droplet size when compared with other experimental uncertainties that must be accounted for (Rovelli et al., 2016).

(b) No uncertainties on the applied density parameterisations are taken into account. However, the sensitivity of the hygroscopic properties retrieved by means of CK-EDB measurements to potential uncertainties in the parameterisation of solution density have been evaluated in Section III.d of the main manuscript. 
Table S2: Measured densities and refractive indices of aminium sulphates solutions.

\begin{tabular}{|c|c|c|c|c|c|c|c|c|c|c|c|c|c|}
\hline \multicolumn{7}{|c|}{ MMAS } & \multicolumn{7}{|c|}{ MEAS } \\
\hline \multirow{2}{*}{$m f s$} & & & \multicolumn{2}{|c|}{$\rho\left(\mathrm{kg} \mathrm{m}^{-3}\right)$} & & \multirow{2}{*}{$\sigma$} & \multirow{2}{*}{$\mathrm{mfs}$} & & & \multicolumn{2}{|c|}{$\rho\left(\mathrm{kg} \mathrm{m}^{-3}\right)$} & \multirow[t]{2}{*}{$m$} & \\
\hline & & & & $\sigma$ & & & & & & & $\sigma$ & & $\sigma$ \\
\hline 0 & \pm & 0 & 998.1 & 0 & 1.333 & - & 0 & \pm & 0 & 998.2 & 0.1 & 1.333 & - \\
\hline 0.0021 & \pm & $8.90 \mathrm{E}-06$ & 999.0 & 0.1 & 1.3334 & - & 0.0073 & \pm & $3.00 \mathrm{E}-05$ & 1000.6 & 0.1 & 1.3342 & - \\
\hline 0.0070 & \pm & $3.00 \mathrm{E}-05$ & 1001.4 & 0.1 & 1.3342 & - & 0.0129 & \pm & $5.30 \mathrm{E}-05$ & 1002.8 & 0.1 & 1.3350 & - \\
\hline 0.0178 & \pm & 0.0001 & 1006.3 & 0.1 & 1.3359 & - & 0.0345 & \pm & 0.0001 & 1010.6 & 0.1 & 1.3382 & $5.77 \mathrm{E}-05$ \\
\hline 0.0406 & \pm & 0.0002 & 1016.8 & 0 & 1.3394 & - & 0.0776 & \pm & 0.0003 & 1026.1 & 0 & 1.3444 & - \\
\hline 0.0675 & \pm & 0.0003 & 1028.8 & 0.1 & 1.3436 & - & 0.1393 & \pm & 0.0006 & 1047.3 & 0.2 & 1.3531 & - \\
\hline 0.1061 & \pm & 0.0005 & 1045.8 & 0.2 & 1.3494 & - & 0.2087 & \pm & 0.0009 & 1071.1 & 0.4 & 1.3632 & 5.77E-05 \\
\hline 0.1487 & \pm & 0.0006 & 1064.8 & 0.1 & 1.3561 & - & 0.3097 & \pm & 0.0013 & 1107.1 & 0.1 & 1.3778 & - \\
\hline 0.1995 & \pm & 0.0009 & 1087 & 0 & 1.3638 & - & 0.4178 & \pm & 0.0017 & 1144.2 & 0.2 & 1.3934 & $1.15 \mathrm{E}-04$ \\
\hline 0.2579 & \pm & 0.0011 & 1111.5 & 0.4 & 1.3724 & - & 0.5295 & \pm & 0.0022 & 1180.6 & 0.2 & 1.4091 & $5.77 \mathrm{E}-05$ \\
\hline 0.3002 & \pm & 0.0013 & 1127.6 & 0.1 & 1.3778 & 5.77E-05 & 0.7687 & \pm & 0.0031 & 1247.6 & 1.2 & 1.4413 & $2.00 \mathrm{E}-04$ \\
\hline 0.3657 & \pm & 0.0016 & 1154.7 & 0.1 & 1.3872 & $5.77 \mathrm{E}-05$ & & & & & & & \\
\hline 0.4411 & \pm & 0.0019 & 1186.8 & 1.2 & 1.3981 & - & & & & & & & \\
\hline 0.5482 & \pm & 0.0023 & 1231.3 & 0.6 & 1.4145 & $1.53 \mathrm{E}-04$ & & & & & & & \\
\hline
\end{tabular}

\begin{tabular}{|c|c|c|c|c|c|c|c|c|c|c|c|c|c|}
\hline \multicolumn{7}{|c|}{ DMAS } & \multicolumn{7}{|l|}{ DEAS } \\
\hline \multirow{2}{*}{$m f s$} & & & \multicolumn{2}{|c|}{$\rho\left(\mathrm{kg} \mathrm{m}^{-3}\right)$} & \multirow[t]{2}{*}{$m$} & \multirow[b]{2}{*}{$\sigma$} & \multirow{2}{*}{$m f s$} & & & \multicolumn{2}{|c|}{$\rho\left(\mathrm{kg} \mathrm{m}^{-3}\right)$} & \multirow[t]{2}{*}{$m$} & \\
\hline & & & & $\sigma$ & & & & & & & $\sigma$ & & $\sigma$ \\
\hline 0 & \pm & 0 & 998.5 & 0.1 & 1.333 & - & 0 & & 0 & 998.1 & 0.1 & 1.333 & - \\
\hline 0.0108 & \pm & 0.0001 & 1001.8 & 0.1 & 1.3345 & - & 0.0024 & \pm & 4.70E-05 & 998.3 & 0.1 & 1.3335 & $5.77 \mathrm{E}-05$ \\
\hline 0.0643 & \pm & 0.0005 & 1019.1 & 0.1 & 1.3421 & - & 0.0124 & \pm & $2.50 \mathrm{E}-04$ & 1000.5 & 0.1 & 1.3347 & $5.77 \mathrm{E}-05$ \\
\hline 0.1232 & \pm & 0.0010 & 1037.6 & 0.1 & 1.3502 & - & 0.0291 & \pm & 0.0006 & 1004.1 & 0.1 & 1.3371 & - \\
\hline 0.1742 & \pm & 0.0015 & 1052.9 & 0.6 & 1.3573 & - & 0.0690 & \pm & 0.0014 & 1012.7 & 0.1 & 1.3426 & - \\
\hline 0.2351 & \pm & 0.0020 & 1071.9 & 0.1 & 1.3657 & $1.53 \mathrm{E}-04$ & 0.1126 & \pm & 0.0022 & 1026.7 & 0.1 & 1.3518 & - \\
\hline 0.2956 & \pm & 0.0025 & 1088.9 & 1.2 & 1.3737 & $2.65 \mathrm{E}-04$ & 0.2094 & \pm & 0.0041 & 1043.3 & 0.1 & 1.3629 & $1.15 \mathrm{E}-04$ \\
\hline 0.3557 & \pm & 0.0031 & 1104.3 & 4.7 & 1.3823 & $2.00 \mathrm{E}-04$ & 0.3061 & \pm & 0.0061 & 1064.9 & 0.1 & 1.3773 & 5.77E-05 \\
\hline 0.4082 & \pm & 0.0035 & 1124.5 & 0.4 & 1.3898 & $5.77 \mathrm{E}-05$ & 0.4292 & \pm & 0.0085 & 1092.2 & 0.1 & 1.3962 & $1.15 \mathrm{E}-04$ \\
\hline 0.4699 & \pm & 0.0040 & 1143.4 & 0.4 & 1.3985 & $5.77 \mathrm{E}-05$ & 0.5622 & \pm & 0.0111 & 1117.3 & 1.4 & 1.4152 & $9.87 \mathrm{E}-04$ \\
\hline 0.5330 & \pm & 0.0045 & 1156.0 & 1.1 & 1.4067 & $1.15 \mathrm{E}-04$ & 0.6667 & \pm & 0.0132 & 1134.5 & 0.2 & 1.4309 & $4.16 \mathrm{E}-04$ \\
\hline
\end{tabular}




\begin{tabular}{|c|c|c|c|c|c|c|c|c|c|c|c|c|}
\hline \multicolumn{7}{|l|}{ TMAS } & \multicolumn{6}{|l|}{ TEAS } \\
\hline \multirow{2}{*}{$m f s$} & & & \multicolumn{2}{|c|}{$\rho\left(\mathrm{kg} \mathrm{m}^{-3}\right)$} & \multirow[t]{2}{*}{$m$} & \multirow[b]{2}{*}{$\sigma$} & \multirow{2}{*}{$m f s$} & & \multicolumn{2}{|c|}{$\rho\left(\mathrm{kg} \mathrm{m}^{-3}\right)$} & \multirow[t]{2}{*}{$m$} & \multirow[b]{2}{*}{$\sigma$} \\
\hline & & & & $\sigma$ & & & & & & $\sigma$ & & \\
\hline 0 & \pm & 0 & 998.0 & 0.1 & 1.333 & & $\overline{0}$ & \pm 0 & 998.0 & 0.2 & 1.333 & \\
\hline 0.0017 & \pm & $1.70 \mathrm{E}-05$ & 998.5 & 0 & 1.3334 & - & 0.0025 & $\pm \quad 3.20 \mathrm{E}-04$ & 998.5 & 0.1 & 1.3336 & - \\
\hline 0.0039 & \pm & $3.70 \mathrm{E}-05$ & 999.2 & 0.1 & 1.3336 & - & 0.0129 & $\pm 7.30 \mathrm{E}-04$ & 1001.3 & 0.1 & 1.3354 & $5.77 \mathrm{E}-05$ \\
\hline 0.0109 & \pm & 0.0001 & 1001.2 & 0.1 & 1.3346 & - & 0.0335 & \pm 0.0012 & 1006.9 & 0.2 & 1.3392 & - \\
\hline 0.0197 & \pm & 0.0002 & 1003.5 & 0.1 & 1.3359 & - & 0.0637 & \pm 0.0016 & 1014.9 & 0.2 & 1.3447 & - \\
\hline 0.0331 & \pm & 0.0003 & 1007.3 & 0.1 & 1.3378 & - & 0.1236 & \pm 0.0023 & 1030.8 & 0.1 & 1.3558 & - \\
\hline 0.0515 & \pm & 0.0005 & 1012.1 & 0.2 & 1.3404 & - & 0.1884 & \pm 0.0028 & 1048.9 & 0.1 & 1.3683 & $1.53 \mathrm{E}-04$ \\
\hline 0.0786 & \pm & 0.0007 & 1019.4 & 0.1 & 1.3444 & - & 0.2747 & \pm 0.0034 & 1075.1 & 0.1 & 1.3864 & - \\
\hline 0.1027 & \pm & 0.0010 & 1025.7 & 0.1 & 1.3477 & $5.77 \mathrm{E}-05$ & 0.3807 & \pm 0.0040 & 1104.8 & 1.2 & 1.4082 & $1.00 \mathrm{E}-04$ \\
\hline 0.1303 & \pm & 0.0012 & 1032.9 & 0.1 & 1.3517 & - & 0.4999 & \pm 0.0046 & 1135.6 & 1.6 & 1.4298 & $1.18 \mathrm{E}-03$ \\
\hline 0.1450 & \pm & 0.0014 & 1036.8 & 0.1 & 1.3539 & $5.77 \mathrm{E}-05$ & 0.6008 & \pm 0.0050 & 1158.2 & 1.8 & 1.4480 & $4.54 \mathrm{E}-03$ \\
\hline 0.1828 & \pm & 0.0017 & 1046.6 & 0.2 & 1.3593 & $5.77 \mathrm{E}-05$ & & & & & & \\
\hline 0.2104 & \pm & 0.0020 & 1053.4 & 0.1 & 1.3634 & - & & & & & & \\
\hline 0.2557 & \pm & 0.0024 & 1065.3 & 0.1 & 1.3701 & - & & & & & & \\
\hline 0.3074 & \pm & 0.0029 & 1078.6 & 0.2 & 1.3778 & $5.77 \mathrm{E}-05$ & & & & & & \\
\hline 0.3638 & \pm & 0.0035 & 1094.0 & 0.2 & 1.3864 & - & & & & & & \\
\hline 0.4580 & \pm & 0.0044 & 1118.1 & 1.6 & 1.4006 & $1.53 \mathrm{E}-04$ & & & & & & \\
\hline
\end{tabular}

Symbols: $m f s$ - mass fraction of solute; $\rho$ - measured density of solution; $m$ - measured refractive index of solution; $\sigma-$ standard deviation arising from the averaging of three repeated measurements. 
Table S3: $3^{\text {rd }}$ order polynomial fitted parameters for densities $(\rho)$, calculated melt densities $\left(\rho_{\text {melt }}\right)$ and melt refractive indices $\left(m_{m e l t}\right)$ calculated with molar refraction mixing rule for the six considered aminium sulphates.

5

10

\begin{tabular}{ccccccc}
\hline & $\boldsymbol{a}_{\mathbf{0}}$ & $\boldsymbol{a}_{\mathbf{1}}$ & $\boldsymbol{a}_{\mathbf{2}}$ & $\boldsymbol{a}_{\mathbf{3}}$ & $\begin{array}{c}\boldsymbol{\rho}_{\text {melt }} \\
\left(\boldsymbol{k g} \boldsymbol{m}^{-3}\right)\end{array}$ & $\boldsymbol{m}_{\text {melt }}$ \\
\hline MMAS & 998.10 & 5.12 & 447.11 & -40.19 & 1410.1 & 1.4771 \\
DMAS & 998.47 & -2.55 & 341.03 & -51.91 & 1285.0 & 1.4725 \\
TMAS & 998.03 & 4.64 & 252.17 & 4.82 & 1259.7 & 1.4921 \\
MEAS & 998.17 & -19.25 & 459.22 & -127.00 & 1311.1 & 1.4708 \\
DEAS & 998.10 & -10.09 & 285.60 & -82.47 & 1191.1 & 1.4810 \\
TEAS & 998.50 & -25.09 & 378.58 & -100.86 & 1251.1 & 1.5357 \\
\hline
\end{tabular}

\section{Parameterisations used:}

$3^{\text {rd }}$ order polynomial parameterization for densities $(\rho)$ as a function of the square-rooted mass fraction $(m f s)$ of solute:

$15 \rho=a_{0}+a_{1}\left(m f s^{0.5}\right)+a_{2}(m f s)+a_{3}\left(m f s^{1.5}\right)$

Eq. S1

Molar refraction mixing rule for the representation of the refractive index $(m)$ of a solution. The molar refraction $(R)$ of a species is defined as (Liu and Daum, 2008):

$R=\frac{\left(m^{2}-1\right) M}{\left(m^{2}+2\right) \rho}$

where $M$ is the compound's molecular mass and $\rho$ is its pure density (melt density in case of a solute, pure liquid density in

20 the case of the solvent, i.e. water). The molar refraction for a mixture of different components is defined as the sum of the molar refractions of each compound $(i)$ weighted by their mole fractions $\left(x_{i}\right)$ :

$R=\sum_{i} x_{i} R_{i}$

Therefore, in the case of a binary solution composed of water and a generic solute:

$\frac{\left(m_{\text {solution }}{ }^{2}-1\right) M_{\text {solution }}}{\left(m_{\text {solution }}{ }^{2}+2\right) \rho_{\text {solution }}}=x_{\mathrm{H}_{2} \mathrm{O}} \mathrm{O} \frac{\left(m_{\left.\mathrm{H}_{2} \mathrm{O}^{2}-1\right) M_{\mathrm{H}_{2} \mathrm{O}}}\right.}{\left(m_{\left.\mathrm{H}_{2} \mathrm{O}^{2}+2\right) \rho_{\mathrm{H}_{2} \mathrm{O}}}\right.}+x_{\text {solute }} \frac{\left(m_{\text {melt }}{ }^{2}-1\right) M_{\text {solute }}}{\left(m_{\text {melt }}{ }^{2}+2\right) \rho_{\text {melt }}}$

Eq. S4

25 where $m_{\text {solution }}$ and $\rho_{\text {solution }}$ are measured, $M_{\text {solution }}$ is calculates as $M_{\text {solution }}=\sum_{i} x_{i} M_{i}$ (Liu and Daum, 2008), the mole fractions of water and solute are known from the concentration of the prepared solution, density and refractive index of pure water are known, $\rho_{m e l t}$ is calculated from the density parameterisation at $m f s=1$. Therefore, the only unknown is $m_{m e l t}$ and a least squares fit of the experimental refractive index data is performed with Eq. S4 to calculate it.

When correcting the obtained radius vs. time data from CK-EDB experiments, the iterative procedure proposed in a previous

30 work (Davies et al., 2012) is applied, but the variation in refracting index with the variation of the solution concentration is calculated with the molar refraction mixing rule. 
Table S4: Comparison of pure melt densities $\left(\rho_{\text {melt }}\right)$ from this work and from Clegg et al. (2013) and solid densities $\left(\rho_{\text {solid }}\right)$ from Qiu and Zhang (2012).

\begin{tabular}{cccc}
\hline Compound & $\begin{array}{c}\boldsymbol{\rho}_{\text {melt }}(\text { this work) } \\
\left(\mathrm{g} \mathrm{cm}^{-3}\right)\end{array}$ & $\begin{array}{c}\boldsymbol{\rho}_{\text {melt }} \\
(\text { Clegg et al., 2013) } \\
\left(\mathrm{g} \mathrm{cm}^{-3}\right)\end{array}$ & $\begin{array}{c}\boldsymbol{\rho}_{\text {solid }} \\
\text { (Qiu and Zhang, 2012) } \\
\left(\mathrm{g} \mathrm{cm}^{-3}\right)\end{array}$ \\
\hline MMAS & 1.410 & $1.451( \pm 0.0029)$ & $1.485 \pm 0.089$ \\
DMAS & 1.285 & $1.278( \pm 0.0022)$ & $1.408 \pm 0.084$ \\
TMAS & 1.259 & $1.289( \pm 0.0031)$ & $1.342 \pm 0.081$ \\
MEAS & 1.311 & - & - \\
DEAS & 1.191 & $1.212( \pm 0.0030)$ & $1.268 \pm 0.076$ \\
TEAS & 1.251 & $1.190( \pm 0.0018)$ & $1.247 \pm 0.075$ \\
\hline
\end{tabular}


Table S5: Values of the coefficients of the fitted equations of measured apparent molar volumes $\left(V^{\phi}\right)$ of aminium sulphates in aqueous solutions (displayed in Figure S3). The used fitting equation is the same used by Clegg et al. (2013): $V^{\phi}=V^{\phi \infty}+a_{1} \cdot(w t \%)^{0.5}+a_{2} \cdot(w t \%)^{0.75} a_{3} \cdot(w t \%)+a_{3} \cdot(w t \%)^{1.5}$

where $V^{\phi \infty}$ is the apparent molar volume of the aminium salt taken from literature (as in Clegg et al.) and $w t \%$ is the weight 5 percentage of salt in solution. Note that the fitted equation is constrained at $w t \%=0$ to the $V^{\phi \infty}$ literature value.

\begin{tabular}{llllllllllllll}
\hline & $\boldsymbol{V}^{\phi \infty}$ & \multicolumn{1}{l}{$\boldsymbol{a}_{\boldsymbol{1}}$} & & \multicolumn{3}{c}{$\boldsymbol{a}_{\mathbf{2}}$} & \multicolumn{3}{c}{$\boldsymbol{a}_{3}$} & \multicolumn{3}{c}{$\boldsymbol{a}_{\boldsymbol{4}}$} \\
\hline MMAS & 86.27 & -0.542 & \pm & 0.276 & & 0 & & 0.792 & \pm & 0.099 & -0.0507 & \pm & 0.00882 \\
DMAS & 123.83 & 2.039 & \pm & 0.150 & & 0 & & & 0 & & 0.00266 & \pm & 0.00367 \\
TMAS & 159.47 & -8.051 & \pm & 0.962 & 6.866 & \pm & 0.883 & -1.318 & \pm & 0.201 & & 0 & \\
MEAS & 120.8 & -1.043 & \pm & 0.238 & 1.062 & \pm & 0.088 & & 0 & & & 0 \\
DEAS & 191.91 & & 0 & & & 0 & & 0.115 & \pm & 0.009 & & 0 \\
TEAS & 256.16 & -22.759 & \pm & 1.264 & 7.080 & \pm & 0.495 & & 0 & & & 0 \\
\hline
\end{tabular}


Table S6: Extended ZSR fitted coefficients (Eq. 3 in Sauerwein et al. (2015)) at two fixed water activities $\left(a_{w}, 0.80\right.$ and 0.925$)$ for mixtures of aminium sulphate salts and sulphuric acid, as in Figure 7 in the main manuscript. The coefficients refer to the ZSR fitting of data from Sauerwein et al. (2016) together with the CK-EDB datapoint (red dashed lines in Figure 7 in the main manuscript). Symbols: $w^{o}{ }_{a}$ - water associated to $\mathrm{H}_{2} \mathrm{SO}_{4}$ in an aqueous solution at the same water activity as the mixture; $w^{o}{ }_{a s}$

5 - water associated to the aminium salt in an aqueous solution at the same water activity as the mixture; $A^{o}$ - fitted empirical parameter.

\begin{tabular}{|c|c|c|c|c|c|c|c|}
\hline & $w_{a}^{o}$ & $w_{a s}^{o}$ & & & $A^{o}$ & & \\
\hline MMAS & 0.2682 & 0.2501 & \pm & 0.0092 & -0.3218 & \pm & 0.0447 \\
\hline DMAS & 0.2682 & 0.3072 & \pm & 0.0047 & -0.4272 & \pm & 0.0240 \\
\hline TMAS & 0.2682 & 0.3782 & \pm & 0.0112 & -0.6147 & \pm & 0.0388 \\
\hline MEAS & 0.2682 & 0.2747 & \pm & 0.0076 & -0.3733 & \pm & 0.0256 \\
\hline DEAS & 0.2682 & 0.3299 & \pm & 0.0195 & -0.4655 & \pm & 0.0882 \\
\hline
\end{tabular}

$a_{w}=0.925$

\begin{tabular}{|c|c|c|c|c|c|c|c|}
\hline & $w_{a}^{o}$ & $w_{a s}^{o}$ & & & $A^{o}$ & & \\
\hline MMAS & 0.5641 & 0.6035 & \pm & 0.0194 & -0.6155 & \pm & 0.0934 \\
\hline DMAS & 0.5641 & 0.6997 & \pm & 0.0248 & -0.7095 & \pm & 0.1273 \\
\hline TMAS & 0.5641 & 0.8143 & \pm & 0.0215 & -1.0166 & \pm & 0.0749 \\
\hline MEAS & 0.5641 & 0.6587 & \pm & 0.0140 & -0.6166 & \pm & 0.0476 \\
\hline DEAS & 0.5641 & 0.8472 & \pm & 0.0325 & -1.0603 & \pm & 0.1233 \\
\hline
\end{tabular}


Figure S1: Schematics of the EDB experimental setup used in this work (view from top).

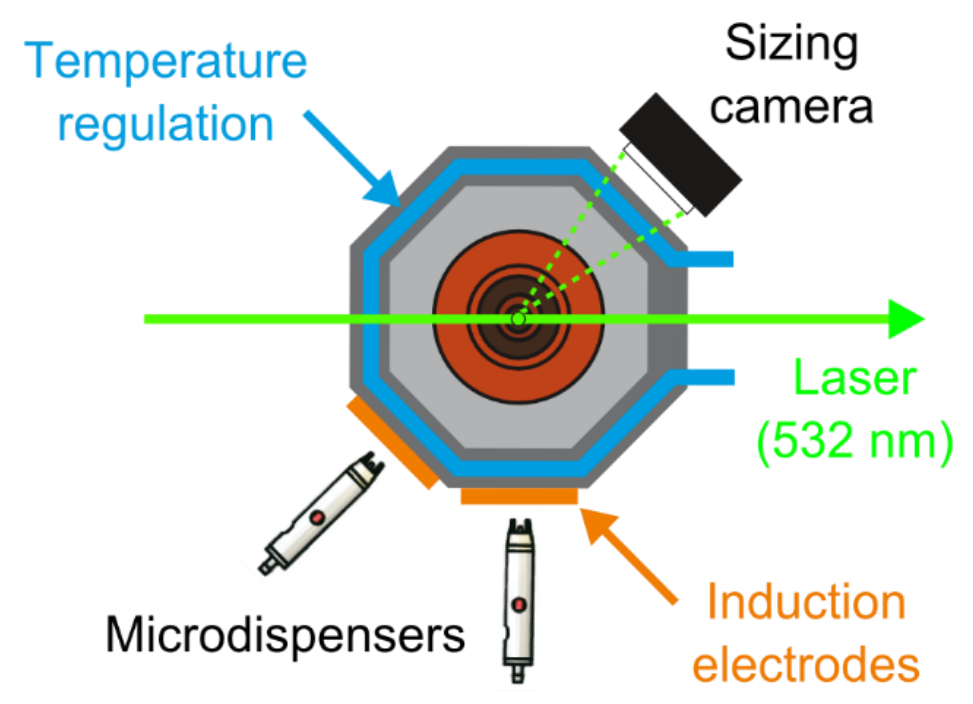


Figure S2: Measured densities of aminium sulphates solutions and $3^{\text {rd }}$ order polynomial fittings of data (Panel (a)). Measured refractive indices as a function of mass fraction and the corresponding molar refraction mixing rule fitting (Panel (b)).
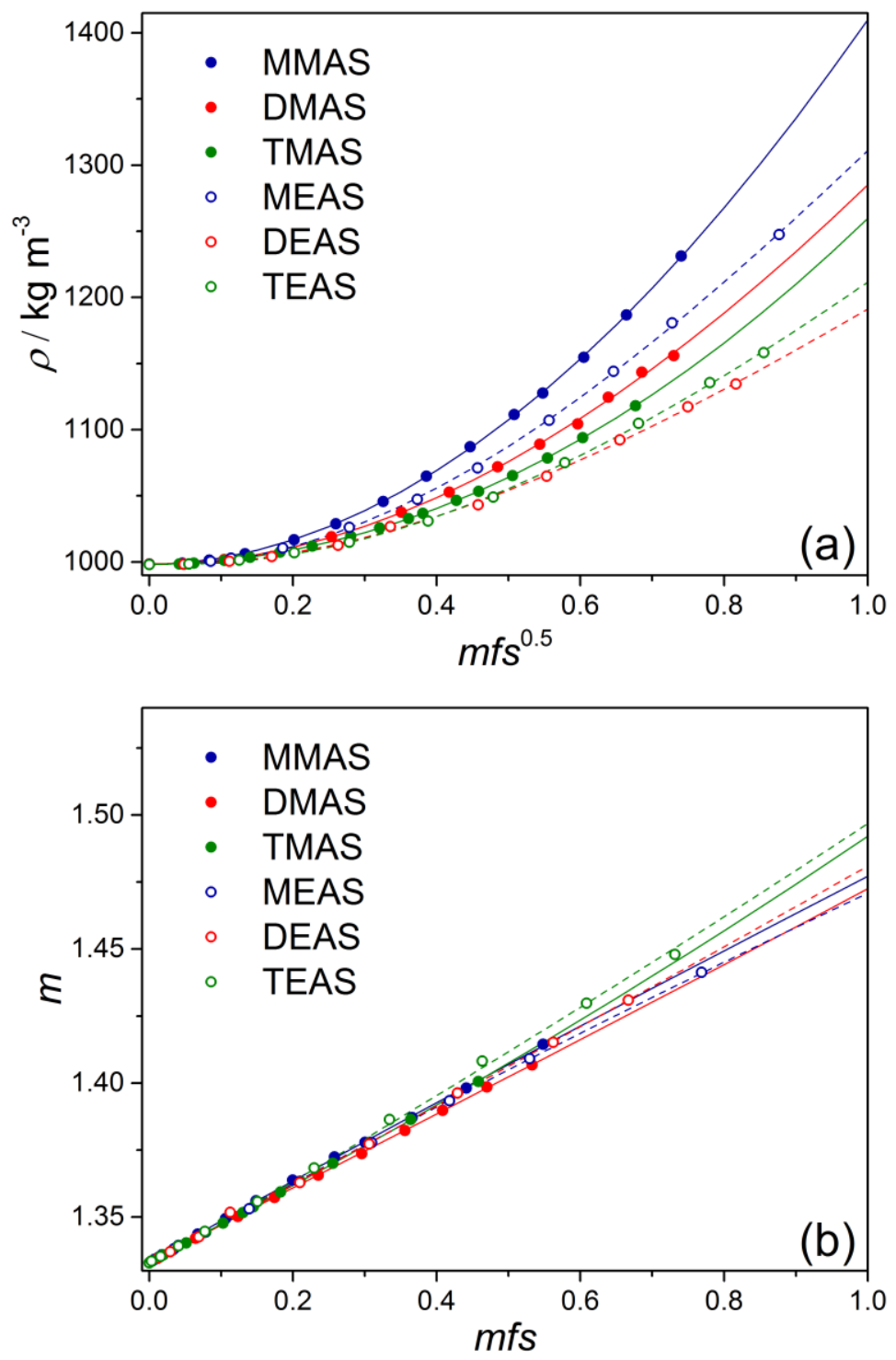
Figure S3: Comparison of measured $G F_{r}$ from CK-EDB experiments and $G F_{r}$ data from H-TDMA measurements by Qiu and Zhang (2012).

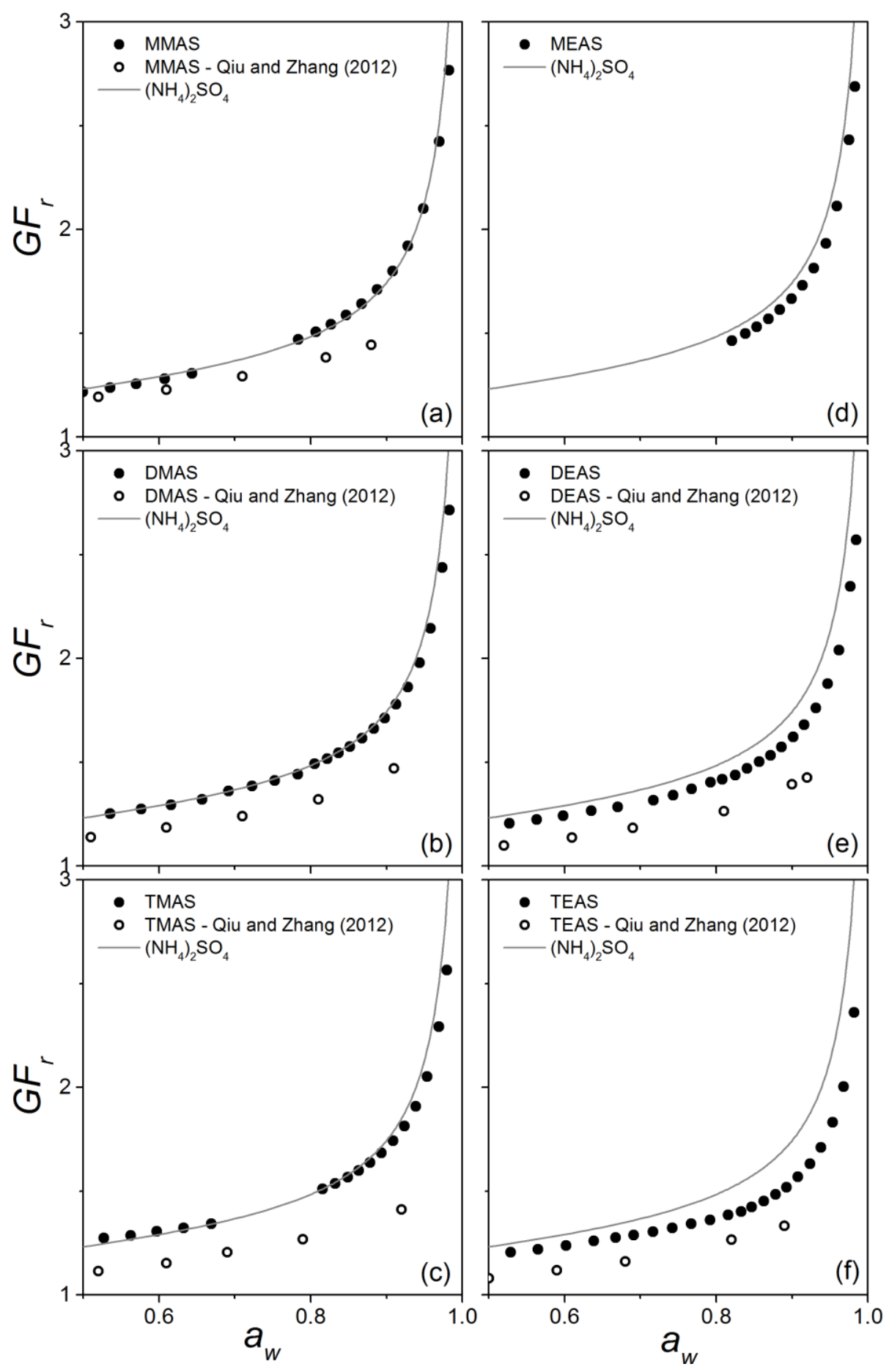


Figure S4: Apparent molar volumes $\left(V^{\phi}\right)$ of aminium sulphates in aqueous solutions as a function of the square root of the solute weight percentage (wt\%). Symbols: black diamonds - measured values, this work; black line - fitted equations (coefficients in Table S5); grey dots - measured values by Clegg et al. (2013); grey lines - fitted equations of mrasured data in Clegg et al. (2013); dashed lines - uncertainty on apparent molar volumes when a $\pm 0.001 \mathrm{~g} \mathrm{~cm}^{-3}$ uncertainty on the measured

5 density is considered; open circles - apparent molar volumes at infinite dilutions from literature (as in Fig. 1 in Clegg et al. (2013)).

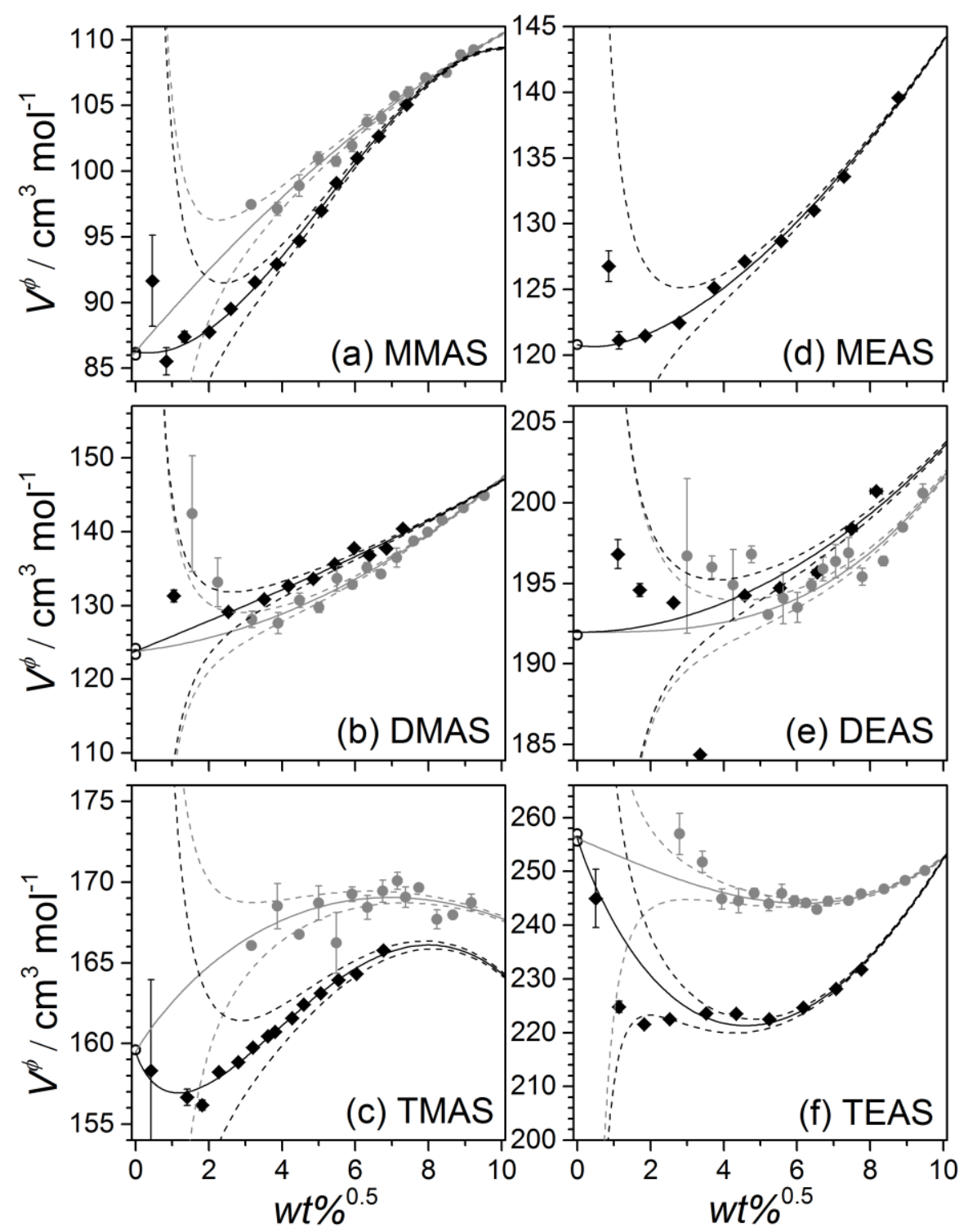




\section{References}

Clegg, S. L., Qiu, C. and Zhang, R.: The deliquescence behaviour, solubilities, and densities of aqueous solutions of five methyl- and ethyl-aminium sulphate salts, Atmos. Environ., 73, 145-158, doi:10.1016/j.atmosenv.2013.02.036, 2013.

5 Davies, J. F.: Characterising The Impact Of Bulk, Surface And Gas-Phase Limitations On Mass Transport In Aerosol., 2014.

Davies, J. F., Haddrell, A. E. and Reid, J. P.: Time-Resolved Measurements of the Evaporation of Volatile Components from Single Aerosol Droplets, Aerosol Sci. Technol., 46(6), 666-677, doi:10.1080/02786826.2011.652750, 2012.

Davies, J. F., Haddrell, A. E., Rickards, A. M. J. and Reid, J. P.: Simultaneous analysis of the equilibrium hygroscopicity and water transport kinetics of liquid aerosol, Anal. Chem., 85(12), 5819-5826, doi:10.1021/ac4005502, 2013.

10 Liu, Y. and Daum, P. H.: Relationship of refractive index to mass density and self-consistency of mixing rules for multicomponent mixtures like ambient aerosols, J. Aerosol Sci., 39(11), 974-986, doi:10.1016/j.jaerosci.2008.06.006, 2008.

Qiu, C. and Zhang, R.: Physiochemical properties of alkylaminium sulfates: Hygroscopicity, thermostability, and density, Environ. Sci. Technol., 46(8), 4474-4480, doi:10.1021/es3004377, 2012.

15 Rovelli, G., Miles, R. E. H., Reid, J. P. and Clegg, S. L.: Accurate Measurements of Aerosol Hygroscopic Growth Over a Wide Range in Relative Humidity, J. Phys. Chem. A, 120, 4376-4388, doi:10.1021/acs.jpca.6b04194, 2016.

Sauerwein, M., Clegg, S. L. and Chan, C. K.: Water Activities and Osmotic Coefficients of Aqueous Solutions of Five Alkylaminium Sulfates and Their Mixtures with H2SO4 at 25oC, Aerosol Sci. Technol., 49(8), 566-579, doi:10.1080/02786826.2015.1043045, 2015. 